\title{
Article
}

\section{Staff experiences and understandings of the REsTRAIN Yourself initiative to minimise the use of physical restraint on mental health wards}

Duxbury, Joy, Thomson, Gillian, Scholes, Amy, Edgar, Fiona, Baker, John, Downe, Soo, Greenwood, Paul, Price, Owen, Whittington, Richard and Mckeown, Michael

Available at https://clok.uclan.ac.uk/25863/

Duxbury, Joy orcid iconORCID: 0000-0002-1772-6874, Thomson, Gillian orcid iconORCID: 0000-0003-3392-8182, Scholes, Amy, Edgar, Fiona, Baker, John, Downe, Soo orcid iconORCID: 0000-0003-2848-2550, Greenwood, Paul, Price, Owen, Whittington, Richard et al (2019) Staff experiences and understandings of the RESTRAIN Yourself initiative to minimise the use of physical restraint on mental health wards. International Journal Of Mental Health Nursing, 28 (4). pp. 845-856. ISSN 1445-8330

It is advisable to refer to the publisher's version if you intend to cite from the work. http://dx.doi.org/10.1111/inm.12577

For more information about UCLan's research in this area go to

http://www.uclan.ac.uk/researchgroups/ and search for <name of research Group>.

For information about Research generally at UCLan please go to http://www.uclan.ac.uk/research/

All outputs in CLoK are protected by Intellectual Property Rights law, including Copyright law. Copyright, IPR and Moral Rights for the works on this site are retained by the individual authors and/or other copyright owners. Terms and conditions for use of this material are defined in the policies page. 


\title{
Staff experiences and understandings of the REsTRAIN Yourself initiative to minimise the use of physical restraint on mental health wards.
}

Duxbury, Joy; Thomson, Gill; Scholes, Amy; Edgar, Fiona; Baker, John; Downe, Soo; Greenwood, Paul; Price, Owen; Whittington, Richard; McKeown, Mick

Accepted for publication: International Journal of Mental Health Nursing

\begin{abstract}
International efforts to minimize coercive practices include the US Six Core Strategies( (6CS). This innovative approach has limited evidence of its effectiveness, with few robustly designed studies, and has not been formally implemented or evaluated in the UK. An adapted version of the 6CS, which we called 'REsTRAIN Yourself' (RY), was devised to suit the UK context and evaluated using mixed methods. RY aimed to reduce the use of physical restraint in mental health inpatient ward settings through training and practice development with whole teams, directly in the ward settings where change is to be implemented and barriers to change overcome. In this paper we present qualitative findings that report on staff perspectives on the impact and value of RY following its implementation. Thirty-six staff participated in semi-structured interviews with data subject to thematic analysis.
\end{abstract}

Eight themes are reported that highlight perceived improvements in every domain of the 6CS after RY had been introduced. Staff reported more positively on their relationships with service users and felt their attitudes towards the use of coercive practices such as restraint were changed; the service as a whole shifted in terms of restraint awareness and reduction; and new policies, procedures and language were introduced despite certain barriers. These findings need to be appreciated in a context wherein substantial reductions in the use of physical restraint was proven possible, largely due to building upon empathic and relational alternatives. However, yet more could be achieved with greater resourcing of inpatient care. 


\section{Introduction}

Events that threaten the safety of patients and staff, such as aggression are not uncommon in mental health inpatient settings (Bowers et al 2015). Staff responses to these incidents can involve the use of practices which restrict an individual and can cause serious physical harm (Paterson et al 2003) and adverse psychological effects (Bonner et al 2002, Rose et al 2015). Restrictive interventions such as physical restraint, seclusion and rapid tranquilisation are used globally (Cowman et al 2017, Bowers et al 2005, 2007, Steinert et al 2010). Coercive practices also include the use of locked wards, rigid cultures, environmental restrictions and many other oppressive features of care (Duxbury \& Whittingham 2005, Cowman et al 2017), having detrimental effects on therapeutic relationships (Stewart et al 2009), which are difficult to repair (Clearly et al 2012b).

The US 'Six Core Strategies@' (6CS) model (Huckshorn 2004, 2006; NASMHPD 1999) for minimising restrictive practices involves:

1. team goals for minimising restraint;

2. systematic reflection on restraint usage;

3. using surveys to better understand aggression on the ward;

4. a partnership approach to reducing restraint;

5. modifying the physical environment and relevant procedures;

6. effective debriefing.

This paper reports upon a qualitative component of a non-randomised controlled trial targeting reducing physical restraint rates. The full multi-method study introduced an adapted version of the Six Core Strategies and evaluated its impact at scale in UK mental health services. The adaptation was based on extensive consultation with stakeholders and became known as 'REsTRAIN YOURSELF' (RY). The aim of the phase of the study reported here was to examine the perspectives of staff regarding the implementation of RY on participating wards following introduction of the intervention. The full implementation process and project evaluation, including secondary outcomes such as the impact on the ward environment and therapeutic relationships, has been more fully, reported upon elsewhere (Duxbury et al 2016).

\section{Background}

Detrimental effects of restrictive practices have been noted for both service users and staff (Duxbury 2015, Gaskin et al. 2007, Huckshorn 2004, O'Brien \& Golding 2003). Research has 
shown that nursing staff consider moral and ethical apects of using physical restraint, often justifying its use as a last resort or necessary evil (Riahi, 2016). In the UK, restrictive interventions attracted increasing attention following associated deaths (Aiken et al 2011) and a particular abuse scandal prompting a government response (DH 2012). Subsequent guidelines, referred to as Positive and Proactive (DH 2014), promoted initiatives and values to minimise the use of such practices.

The 'Six Core Strategies' for minimising seclusion and restraint is a multilevel complex intervention targeting both organisational and individual factors in decision-making about care in acute mental health services (LeBel et al 2014). The underpinning training curriculum to reduce and prevent the use of seclusion and restraint is a comprehensive approach for practice and culture change developed by the National Association of State Mental Health Program Directors (NASMHPD) and Huckshorn, (2004; 2006; Huckshorn \& LeBel, 2009; NASMHPD, 2011). The implementation of programmes informed by the Six Core Strategies approach has been associated with reduced seclusion and restraint in a number of North American evaluations (Barton et al., 2009; LeBel, 2011; Lewis et al 2009; Wieman et al., 2014, Riahi et al., 2016, Goulet et al 2017). There is also evidence of broader improvements for service users and staff including greater satisfaction with care, reduced injuries, decreased medication use, shorter admission duration and reduced staff turnover and absenteeism (Barton et al., 2009; Paxton, 2009; LeBel, 2011; Sanders, 2009, NICE 2015, DH 2014).

Consequent benefits of the reduced use of restraint for service users have been identified including increased satisfaction with care (LeBel, 2011; Murphy \& Bennington-Davis, 2005), reduced injuries and length of stay (Thomann, 2009), decreased medications (Barton et al., 2009; Smith et al., 2008), and reduced recidivism (Paxton, 2009). Other benefits include reductions in staff turnover and related costs (Regan et al 2006; Paxton, 2009), decrease in staff injuries, absenteeism, hiring, and retraining costs (LeBel, 2011; Murphy \& BenningtonDavis, 2005), and increased staff satisfaction and retention (Regan et al., 2006; Sanders, 2009).

A number of restraint minimisation programmes include 'Safewards' originating in the UK (Bowers 2014), and 'No Force First' (Ashcraft \& Anthony 2008) and the 'Engagement Model' (Borckardt et al. 2011) from the US. The Safewards approach has been shown to effectively reduce use of restrictive practices in a number of national contexts (Bowers et al 2015; Fletcher et al. 2017). A systematic review demonstrating the value of such remedial programmes notes that most relevant evidence is furnished by adaptations of the $6 \mathrm{CS}$ approach (Goulet et al. 2017), which has been successfully adapted for European mental 
health services (Guzman-Parra et al. 2016; Putkonen et al. 2013). A recent Australian survey investigated nurses' perceptions regarding reducing and eliminating restrictive practices. These were viewed as necessary last resort methods to maintain staff and consumer safety, and nurses tended to disagree that containment methods could be eliminated from practice. They also felt that containment use was a function of a lack of resources (Gerace \& Muir Cochrane 2018).

The wider evaluation of the RY project demonstrated the value of the adapted 6CS approach in this national context, with a reduction in the use of restraint on the intervention wards of on average $22 \%$, with a $60 \%$ reduction on some wards (Duxbury et al submitted). An exploration of staff views on an adapted approach to minimise the use of restraint practices continues to be important in understanding relevant process issues in any implementation.

\section{Methods}

This qualitative inquiry was embedded in a larger study aiming to evaluate the implementation of RY using a mixed-methods, non-randomised controlled trial design (Duxbury et al 2016). The overarching evaluation comprised a mixture of qualitative and quantitative approaches including the measurement of physical restraint rates, the outcome of staff and patient surveys and interviews and ethnographic data.

Fourteen acute mental health wards in the North West of England took part, with seven receiving the $R Y$ intervention and seven served as controls. Whilst impact evaluation in the form of pre and post-test measures is essential to establish whether interventions work, this does not address questions of 'why' and 'how', nor examine relationships between inputs and outcomes (Stame, 2004). Analysis of post-intervention interview data undertaken with staff on the intervention wards to explore staff perspectives and experiences of the implementation of the RY approach is presented here. There was some attempt to purposively seek as diverse a sample as possible (in terms of role, grade, shift pattern, gender, ethnicity), but stratification was not attempted, and the participants are best described as a convenience sample. The interviews followed a topic guide designed to explore experiences of implementation and any barriers to change. The topic guide was not structured using the 6CS headings to avoid leading responses. Four members of the research team (initials here) conducted the interviews in private spaces within the respective wards. The researchers conducting interviews comprised research assistants with a psychology background (initials) and with substantial experience as a service user (initials), 
supported by senior researchers, a mental health nurse by background (initials) and mental health focused social scientist (initials). Ethic approval was granted by the NHS Health Research Authority (ref 14/YH/0164)

RY adapted the 6CS approach by revising language and generally modifying to suit the UK context. Over the period of the project, and within all the participating Trusts, a range of different innovations were rolled out on the implementation wards within a six core strategy framework. The RY intervention involved initial training relating to the $6 \mathrm{CS}$ approach, away from the ward environment. This introduced the ethos and purpose of the project, encouraging reflection upon issues to be targeted throughout the six-month implementation period. This was followed by a series of stages involving intensive, facilitated ward based learning for the whole team and adoption of new ways of working. Continuous feedback was sought regarding success of planned actions, challenges and learning points. This enabled adjustment of plans, development of new procedures, and refinement of the training, support, and supervision provided to meet specific ward contingencies whilst maintaining a focus upon the 6CS.

To prepare the participating ward teams a 'Train the Trainer' model was used to roll out training. An online toolkit, incorporating theory, Quality Improvement methodological techniques, case examples and top tips, was developed to support the implementation of the approach. (https://www.aquanw.nhs.uk/resources/restrain-yourself-toolkit/20917). Local steering groups were set up to progress ongoing spread and sustainability. The approach focused on identified champions for each ward and attendance at action learning sessions on a monthly basis.

A dedicated improvement adviser worked on the wards one day a week to support the implementation of the approach, emphasising:

- The identification of relevant change ideas with staff.

- Use of Plan, Do, See, Act cycles to enact change.

- The identification of group and individual roles and ownership, whereby each member of the team commits to individual actions to achieve agreed goals.

Following the implementation phase, audio-taped semi-structured interviews were conducted with 36 staff, to identify changes in practices and attitudes. Data were organised using MAXQDA (version 11) and subject to thematic analysis (Braun \& Clark 2005). The analysis drew upon the elements of the 6CS to construct a coding frame, allowing for thematic 
consideration of data relevant to staff views associated with salient goals. The analysis was completed by the four team members involved in data collection. Two researchers (initials) independently coded a number of the interviews, discussing analytical decisions and refining the coding frame. Identified themes were shared and agreed in a process of deliberative discussion by all four researchers.

\section{Findings}

Eight themes were identified, which are summarised below with illustrative quoted material. The themes are: leadership and impetus towards organisational change; data to inform practice; meaningful activities and events; changing hearts and minds; use of restraint reduction tools; patient voice and agency; debriefing techniques; and, embedding and sustaining developments. Cross-cutting concerns regarding the influence of staffing levels and deployment of bank and agency staff were associated with many of the thematic accounts. This was felt to be of sufficient importance to merit substantive attention in its own right and a more complete discussion of the impact of staffing resources is presented in a companion paper (authors, submitted). In short, perceived inadequacies in staffing were felt to adversely affect levels of use of restrictive practices and attempts to remedy this.

\section{The impetus for organisational change}

This account details efforts for creating impetus for positive change, with leadership being an important component in this regard. Perceived deficits in the process that might impede such change, including lack of institutional support, and potential for counter-intuitive practices are also referred to.

Many staff members had positive experiences of the training, acknowledging the initial impetus for organisational change. Some healthcare professionals reported how the information and messages conveyed through the training session (which included service users discussing their experiences of restraint) were a catalyst for deeper reflection on practice, values, and ward culture. On a number of occasions, staff remarked how scenarios and discussions within the training were felt to be beneficial for real-life application in the wards. The combination of having an opportunity to learn as a team and bring their own experiential knowledge to the learning process was appreciated, and inspired commitment to application: 
So just the fact that the team was together, and it was given a lot of importance [...] we discussed examples of things that had happened, we were able to all comment ... Because it was given time and obvious resource and commitment from several people, this was really something we were going to go ahead and pilot. And it was our ward and this is what we're going to do (Site 1_Staff 1_T2)

A number of staff, however, were negatively disposed to the learning process and its potential to support change, feeling there was too much focus in some sessions on data and graphs, rather than people, or the means for practice-based changes. Others felt that the training was overly didactic, offering few new insights, or felt like unfounded criticism of their professionalism:

It was like teaching your grandma to suck eggs [...] a lot of us felt insulted because you're telling us how to do a job and if we were bad at our job, l'd understand it, but I don't think we are (Site 5_Staff 1_T2)

A key component of the 6CS initiative is 'clear executive leadership' (Huckshorn, 2015), integral to any efforts to promote organisational change. In our study, the fact that senior management were visibly involved was considered to be key in the success of the project. However, in a number of wards inconsistent management or individual leadership steering the project meant that implementation of some of the RY strategies was limited:

There's been no clear figurehead and sort of lack of continuity I think ... rather than sort of looking ahead and planning with the staff (Site 2_ Staff 12)

A number of staff reported how management seemed disconnected from the ward culture, lacked visibility at ward level, or were unresponsive to events:

I think management's very poor, to be honest. I think there's a lot of flaws within the management system ... I've worked on several wards and l've never known one like 
this, to be honest. And I think it's just slowly but surely getting worse (Site 7_ Staff 2_T2)

Furthermore, some staff perceived that management directions undermined the aim of reducing restraint:

We've been told that because of the unpredictability and the risk and the kind of escalating behaviours, that we need to be a bit more forceful (Site 7_ Staff 2_T2)

\section{Data to inform practice}

The value of reflecting on data regarding incidents of violence and aggression and restraint practices was reported. Overall, the majority of staff were unaware of such practice, or it had been infrequently undertaken. However, for others, the opportunity to review restraint figures was considered a useful aid for reflection, as well as providing positive feedback for the team:

There's more awareness and thought ... and the recording of things and people actually thinking, well what's gone on. Whereas now they're looking at it as a whole picture (Site 4_Staff 7_T2)

Safety Crosses were considered to be an accessible means to raise awareness of restraint use. These are a visual aid for recording incidents over a monthly period, indicating dates any restraint or violent incident occurred, displayed in the staff office for regular review. Staff felt this tool benefited team cohesion, enabling reflections on ward climate and successful practices. More critical views saw this tool as an additional burden on staff time, detracting from patient contact, or its use tailing off once support in the RY implementation phase was withdrawn. There was similar critique of the relevance or achievability of restraint reduction due to the unpredictable nature of admissions and levels of acuity:

Restraint is a last resort, so no one ever aims to set out to achieve this many or that many (Site 2_Staff 14_T2) 


\section{Meaningful activities and events}

Attempts to introduce meaningful activities for service users were initiated aiming to foster collaboration with staff, alleviate boredom and frustration, and support therapeutic relations. This involved three particular initiatives: 'protected meal times'; 'visible nurse' and 'needs-led events'.

As well as efforts to enhance the experience of mealtimes (for example, patients creating their own tablemats and playing music) staff made reference to the value of dining together in strengthening therapeutic relationships. This, however, proved controversial. Management resisted allowing staff to eat hospital food, and even when some wards acquired funds to facilitate this, staff willingness was variable. In some wards, a compromise of staff eating their own food was introduced.

Usual practice on the wards was for healthcare assistants to be available to patients. However, their level of authority constrains responsiveness to patient demands (concerning matters of leave, medication, acquiring personal belongings) so patients were seen to congregate outside staff offices in order for requests to be resolved; leading to increased frustration and potential for conflict. Efforts to ameliorate this included increasing the visibility of registered nursing staff. The intention was for a nurse on each shift to assume responsibility for spending prolonged periods of time in communal areas and being a point of contact for patients. However, in reality, this proved challenging to organise consistently because of poor staffing levels.

Excursions were more frequently organised, with more varied, socially inclusive and ageappropriate options; also freeing up quality time for those remaining on the wards:

We were doing like little trips out to like the museum and stuff ... two of us would go out and we'd take like four or five patients. And it means that like the ward's quieter and calmer and those patients, you know, have done something nice. (Site 3_Staff 1_T2) 
As a direct result of RY all of the intervention wards reported increases in activities as well as staff flexibility and willingness to provide these, 'playing to people's strengths' in assigning staff members to lead activities that matched their interests.

\section{Changing hearts and minds}

The RY intervention was reported to have had a positive influence on the beliefs, attitudes and behaviours of staff, enhancing care, delaying intent to restrain and increasing understanding of trauma. Staff reported changing attitudes and behaviours in responding to and engaging with patients. Rather than just perceiving service users as a 'patient' or a diagnosis, they referred to adopting a more individualistic approach to create a therapeutic environment. Unsurprisingly, the majority of staff held negative attitudes towards the use of, and impact of restraint. While some staff considered physical restraint to be a 'necessary evil', this practice created fear, anxiety and trauma for all concerned, including damage to staff-patient relationships. Some staff reflected on how before the RY project, restraint could be 'used too soon' or to 'teach a lesson'.

Staff reflected on specific cases resonant with principles of practicing in a least restrictive manner. In these circumstances, increased knowledge and awareness of the impact of restraint led staff to display less threatening interpersonal behaviours. They believed that they had become more compassionate and understanding of patient behaviours:

We take a step back, we talk to them and recognise that, in some cases, people have to, it's their way of getting rid of whatever's inside them (Site 1_Staff 11_T2)

Some described how a more consistent team approach had been adopted in managing violent and aggressive behaviours and how they were more confident in using verbal deescalation and supporting distressed patients: 
I've learnt over the last few months ... think to myself, somebody hasn't just kicked off because they're kicking off. There is a reason around it. (Site 1_Staff 11_T2)

While a few staff considered they had always practised this way, others believed the RY project had helped re-define when to intervene:

We always used to use restraint as a last resort but I think we always used to think we did, rather than actually do it. And I think in our heart of hearts we believed that. But I think now, being involved in this project, that actually, now we're doing that and there has been a shift (Site 1_Staff 3_T2)

Other views about changes in ways of responding enabled staff to have 'more time to talk to patients' and 'to do my job', with increased job satisfaction, which in turn had salutary outcomes for patients (such as helping staff identify and respond to patient triggers for violence and aggression).

In response to identified need, an element of the RY training was designed to introduce helpful strategies when caring for individuals diagnosed with personality disorder. This was co-produced and facilitated by a service user who also carried that diagnostic label. Staff referred to how this training enhanced understanding of behaviour such as self-harm and how responses could exacerbate patient's negative internal schemas. Staff referred to how this knowledge and awareness had precipitated more 'mindful' and 'empathic' engagement with patients:

Somebody's not cutting themselves just to annoy you ... I've never been through the trauma that they've been through (Site 5_Staff 8_T2).

And that's the difference now I think, and to actually understand that if we do lay hands on, we might actually be doing more damage (Site 3_Staff 8_T2) 
Others recounted how the training had promoted safer self-harm strategies rather than attempts to eliminate these behaviours. However, in a number of instances, these were blocked by risk-aversive medical staff. Furthermore, some staff retained pejorative attitudes to individuals' deemed personality disordered.

\section{Use of restraint reduction tools}

A number of approaches aligned to minimising restraint, supported with specific tools or processes, were developed, including engaging service users in co-producing safety, and efforts to enhance the therapeutic potential of the setting.

Safety plans were introduced as part of the project to help identify risk factors for violence and aggression. These are a self-management tool for service users to identify and record personal triggers, early warning signs and calming strategies in relation to aggression. Mixed views were offered on their introduction; from 'empowering patient control' to concerns about 'suitability for all' and 'validity of use'. From a positive perspective, safety plans were seen to embody person-centred care, empowering patients to take 'control' and 'ownership' in communication:

Helping the patient make their own choices and make them feel like they've got options (Site 3_Staff 5_T2).

Safety plans were also seen as an important means to communicate and raise awareness amongst staff about how to respond to individual patients. Overall, however, introduction of this tool was inconsistent. Some wards appeared to have adopted the plans into practice, but often only for certain patients, and being utilised by particular staff. Some staff were not aware of the use of safety plans; 'I don't think we've done any of them' (Site 5_Staff 20_T2), and others reflected on more haphazard adoption, which could, in part, be due to the levels of challenging behaviour experienced. One noted difficulty in using safety plans was incompatibility with electronic record-keeping systems.

Wards were often described in non-therapeutic terms. Participants complained about the level of noise on the units, the lack of space and poor interior design features. While part of 
the RY intervention was to introduce comfort or sensory rooms, this only occurred on one ward. Staff described this as an 'amazing resource' that was well utilised, providing a 'sacred space' to improve patient's emotional and spiritual wellbeing:

It certainly calmed him down, he was there for two or three hours just chilling, listening to music. I mean never once did it ever come to a restraint and that room has definitely, definitely helped (Site 3_Staff 5_T2)

\section{Patient voice and agency}

Various strategies for patient empowerment and involvement were reported, including recognition of formal and informal peer support. One RY strategy involved introduction of community meetings. These involved opportunities for patients to: come together to discuss ward-based issues; report a sense of safety; communicate and plan activities; encourage support seeking; engage service users in a social forum; and explore ways to improve patient experiences. On one of the wards, a suggestion box was introduced, together with a notice-board offering a valuable visual acknowledgement of patients' views and noting appropriate action was underway. While some wards had a history of ad-hoc patient forums, staff referred to RY providing additional impetus for such meetings. Conversely, staffing or patient acuity levels could lead to cancelled meetings. There were also frustrations for staff unable to implement patient-led changes due to resource or management restrictions.

Staff also referred to the use and utility of weather symbols aiding patients to describe the ward atmosphere:

if it's been stormy, has there been any arguments or anybody feeling like they've been like bullied in any way, or if it's been sunny ... And if it's anybody saying, it's been a bit cloudy, then that's an opportunity. I say, well, is there anything in particular that's made you feel like that? (Site 4_Staff 8_T2)

There was some evidence of positive informal support between patients. The value of these relationships was acknowledged by staff, particularly when a lack of time restricted their own 
opportunities for direct patient contact. However, it was noted some service users reported that providing support to others could be detrimental to personal wellbeing.

\section{Debriefing Techniques}

Central to the $6 \mathrm{CS}$ is debriefing of incidents. Some staff reflected on how the RY project had created a cultural shift in debriefing practices whilst recognising challenges. Whereas other staff felt there had been no changes due to an already established model of practice. However, more frequently reported was an embedding of debriefing within practice and positive consequences for staff and service users. From this perspective, previous debriefing practice had been purely to assess immediate welfare. Following training in trauma informed care, debriefing became viewed as an invaluable opportunity to employ root cause analysis; to learn more about patients' needs and triggers, and how to collaboratively address future circumstances:

She said she'd been raped when she was younger and that she didn't like people grabbing her. She identified two staff that were very heavy handed with her ... And, you know, looking at what I seen the other day, was someone that had completely recovered, and she recognised that as well. So, obviously, something has worked. (Site 3_Staff 5_T2)

The routine use of debriefing, formal recording of the sessions, and its impact was described as a 'massive cultural shift'. Participants, however, noted barriers to routine adoption of debriefing, including lack of time, patient acuity and poor staffing levels. Also, debriefing was felt to be unsuitable for certain patients or there was a tendency to debrief for less serious events rather than 'severe' incidents of violence and aggression. Despite efforts to implement a mandatory process of debriefing for staff and service users, some of the professionals interviewed were unaware of any changes within practice.

\section{Embedding and sustaining developments}

Given appreciation for practices resulting from the RY implementation, participants commented on the importance for change to endure beyond the project end: 
For me, one of the biggest things is about sustainability ... without a doubt, l've seen positive results. But it's about ensuring that is sustained and embed it in practice and that actually, when you guys all go away and we don't have that involvement, that within six months we don't go back to how we were (Site 1_Staff 3_T2)

A number of sustainability strategies were suggested to help ensure staff remained informed of the purpose and value of new approaches and enduring impact on ward culture. One suggestion was for prolonged auditing processes to assess on-going utilisation of RY techniques. Other staff wanted additional training days for reflection and 'regrouping', ensuring continual learning and skills development. There were also calls for posters in staff areas toreinforce the techniques and ethos of RY.

\section{Discussion}

This presentation of the qualitative experiences and understandings of staff involved in the implementation of RY further strengthens consideration of the feasibility of adopting such approaches. Our observations on the challenges faced within this particular change process are not unique to these settings (Bevan \& Fairman 2014, Grol et al 2013, Robert et al 2015), or indeed other mental health care change initiatives (Janner 2007, Woltmann et al. 2008). These findings chime with extant evaluations of the 6CS, and other restraint minimisation approaches such as Safewards (Bowers 2014, Bowers et al 2015) and No Force First (Ashcraft \& Anthony 2008).

The participatory nature of the RY implementation arguably served to allay many of the fears of staff, improving attitudes and beliefs about restraint reduction. Broader findings and the accounts expressed here demonstrate restraint rates can be reduced and relationships and practices improved. However, these developments can only be maintained if there are clear strategies to sustain the innovation and disseminate learning. Moreover, the interview data illustrates that the contemporary context framing the implementation and evaluation of RY presented various challenges which need to be understood and addressed if potential benefits are to be optimised. As for any transformational process, enthusiasm, commitment and engagement of participants was crucial, together with managerial support and leadership (Aarons et al. 2017, Corrigan et al. 2002). Different levels of managerial support were remarked upon; some teams facing contradictory organisational expectations, with frustrated desires for dynamic leadership and management. In the extreme, front-line staff 
could not trust that institutional commitment to change ideals was actually forthcoming, stable, or likely to endure. The potential for some staff to be offended by perceiving an implicit criticism of their previous use of restraint, especially in a context of denuded resources and staffing levels, has to be sensitively dealt with in a context of demonstrating potential benefits. The perception that lack of resources are implicated in levels of coercion and undermine efforts at reduction is reinforced in other studies of staff views (Gerace \& Muir Cochrane 2018).

Overall, the benefits of the RY intervention, achieved through introduction (or reinvigoration) of particular tools and techniques, were believed to have created positive individual, interpersonal, environmental and cultural changes. This included whole team collaboration and motivation to engage in reflective practices for harm minimization. As reported by others (e.g. Janner, 2007), meaningful, flexible needs-led activities and events promoted therapeutic staff-patient relationships and engagement of service users in positive pursuits. The use of safety plans and more regular use of debriefing also meant that staff members improved understanding of the difficulties and challenges faced by service users, particularly those carrying a personality disorder diagnosis and seen as challenging in the ward context. This was congruent with increasing acceptance of an ethos of trauma-informed care (Muskett 2014, Sweeney et al. 2016).

Health professionals referred to adopting a more person-centred and compassionate approach to care. They related being more mindful of not displaying threatening interpersonal behaviours, stepping-back and observing behaviours rather than operating a knee-jerk, interventionist approach. Commitment to a more consistent team approach in managing violent and aggressive behaviours, and increased confidence in using verbal deescalation is also reflected in other restraint minimisation interventions (Price \& Baker 2012, Price et al. 2015). An impetus to create positive environmental changes to the ward environment was evident, such as through patient involvement in the re-design of patient areas; now an emergent focus of service user involvement (Curtis et al. 2007). Service user meetings created a space for discussion and sharing, issues, contributing to ward improvements and positive relationships with staff and peers, confirming other studies of involvement and empowerment (Tambuyzer \& Audenhove 2015). 
Staff critics of the initiative considered new practices as unsuitable for certain patients.

These staff felt disempowered in the process and perceived additional burdens on their time. Concerns about colleagues lacking skills for practising de-escalation were raised, as well as the reticence or capacity of staff and patients to engage in particular activities or community meetings. However, the most prevalent and pervasive issue was the lack of staff to facilitate and implement core implementation activities. Low staffing levels, the burden of non-clinical, administrative tasks and the chaotic and unpredictable nature of the ward climate meant that capacity to facilitate progressive practices was curtailed, particularly when patient acuity was high. A further challenge was the perceived false economy of over-reliance on bank and agency staff - a practice employed by all the Trusts involved. The temporary employment of staff without prior relationship with patients or knowledge of the harm minimization approach potentiated a lack of fidelity in embedding new implementation approaches, resonating with previous critiques of the deleterious impacts and uncertainties consequential on absence of regular staff (Bauman 2000, Bowers et al. 2007).

One success of our project was that it moved beyond simple description of complicating and constraining factors towards insights into applications within contemporary practice. The positive impacts noted are testimony to ways in which these issues were foregrounded in the training interventions. Having the opportunity to reconnect with compassionate and humane role motivations in a public but protected space may have contributed to the positive changes noted in many wards in the post-implementation period. Evidence of better understandings of the nature and experience of the contested notions of personality disorder and self-harm from the service user perspective was a particular example of a departure from 'othering' (Wright et al 2007), and over-use of restraint applied to these groups (Brophy et al. 2016).

Despite the reported successes, coercion within mental health services remains powerfully present and is maintained by pervasive societal governance factors privileging a focus on risk, that individual nurses and teams have little influence over (Beck 1992, Coffey et al 2017, Lupton 1993, Rose 1990). Gerace and Muir Cochrane (2018) show that whilst factors perceived to reduce the likelihood of restraint include the use of empathy, rapport between staff and service users and utilizing trauma-informed care principles, nurses are faced with threatening situations and feel only moderately safe at work. This means that initiatives at multiple levels are needed to help nurses to maintain safety and move towards realizing directives to reduce and, where possible, eliminate restraint use. Importantly, in our study we 
found that staff had to gain faith in the instrumental value of alternative approaches to volatile and disturbed individuals, or in the context of self-harm. They had to come to believe that acting differently would actually work in managing or calming the situation. They also had to be persuaded that doing different things as a team would be allowed within prevailing rule-laden governance systems; believing changing practice would be managerially supported and, indeed, legitimated.

\section{Conclusion}

Here, we have described a range of staff experiences, appreciation and understandings of the implementation of RY. The fact that, despite counter-vailing forces, RY was able to make progressive inroads into reducing instances of restraint and altering various ward practices is an endorsement of the RY approach. In many regards, to begin with, circumstances within acute mental health units was inopportune for anticipating such a positive impact.

These qualitative findings assist in making sense of the demonstrable reductions in use of restraint and changes to the psychosocial ward environment in the course of the RY project. Arguably, these outcomes are best explained with recourse to the participatory, relational characteristics of the intervention, which enabled teams of staff to take collective ownership of changes in their own ward environs, and to build positive engagement with service users through empathic identification. With additional investment in overall resources and managerial support even better outcomes might be possible in the future. Arguably, the need for minimisation of physical restraint is as urgent as ever given the prevalence and significance of injurious consequences.

\section{Implications for Practice}

Despite certain reservations, the RY approach to restraint reduction was a success from a staff perspective. Each of the six strategies were welcomed and used in practice. Hence, $\mathrm{RY}$ can be implemented successfully with support of staff who have confidence in the organisational approach. Adequate resources are essential for maintaining staff commitment and sustaining implementation, such that patient care is enhanced and potential for physical and psychological harm is minimised. 


\section{References}

Aarons, G.A., Ehrhart, M.G., Farahnak, L.R., Sklar, M. and Horowitz, J. (2017).

Discrepancies in leader and follower ratings of transformational leadership: Relationship with organizational culture in mental health. Administration and Policy in Mental Health and Mental Health Services Research, 44(4), 480-491.

Ashcraft, L. and Anthony, W. (2008). Eliminating seclusion and restraint in recovery-oriented crisis services. Psychiatric Services, 59: 1198-1202.

Barton, S. A., Johnson, M. R., Price, L. V. (2009). Achieving restraint-free on an inpatient behavioral health unit. Journal of Psychosocial Nursing and Mental Health Services, 47: 34 40.

Bauman, Z. (2000). Liquid modernity. Cambridge: Polity.

Beck, U., (1992). Risk society: Towards a new modernity. London: Sage.

Bevan, H. and Fairman, S. (2014). The new era of thinking and practice in change transformation: a call to action for leaders of health and care, London: NHSIQ (NHS Improving Quality).

Borckardt, J.J., Madan, A., Grubaugh, A.L., Danielson, C.K., Pelic, C.G., Hardesty, S.J., Hanson, R., Herbert, J., Cooney, H., Benson, A. and Frueh, B.C. (2011). Systematic investigation of initiatives to reduce seclusion and restraint in a state psychiatric hospital. Psychiatric Services, 62(5), 477-483.

Bowers, L. (2014). Safewards: a new model of conflict and containment on psychiatric wards. Journal of Psychiatric and Mental Health Nursing, 21(6), 499-508.

Bowers, L., Allan, T., Simpson, A., Nijman, H. and Warren, J. (2007). Adverse incidents, patient flow and nursing workforce variables on acute psychiatric wards: The Tompkins Acute Ward Study. International Journal of Social Psychiatry, 53(1), 75-84.

Bowers, L., James, K., Quirk, A., Simpson, A., Stewart, D. and Hodsoll, J. (2015). Reducing conflict and containment rates on acute psychiatric wards: The Safewards cluster randomised controlled trial. International Journal of Nursing Studies, 52(9), 1412-1422.

Braun, V. and Clarke, V. (2006). Using thematic analysis in psychology. Qualitative research in Psychology, 3(2), 77-101.

Brophy, L.M., Roper, C.E., Hamilton, B.E., Tellez, J.J. and McSherry, B.M. (2016).

Consumers and their supporters' perspectives on poor practice and the use of seclusion and 
restraint in mental health settings: results from Australian focus groups. International journal of Mental Health Systems, 10(1), 6.

Campbell, D. (2018). Figures reveal 'alarming' rise in injuries at mental health units. The Observer, https://www.theguardian.com/society/2018/jun/09/nhs-restraint-techniquesmental-heath-patient-injuries-rise

Coffey, M., Cohen, R., Faulkner, A., Hannigan, B., Simpson, A. and Barlow, S. (2017). Ordinary risks and accepted fictions: how contrasting and competing priorities work in risk assessment and mental health care planning. Health Expectations, 20(3), 471-483.

Corrigan, P.W., Diwan, S., Campion, J. and Rashid, F. (2002). Transformational leadership and the mental health team. Administration and Policy in Mental Health and Mental Health Services Research, 30(2), 97-108.

Curtis, S., Gesler, W., Fabian, K., Francis, S. and Priebe, S. (2007). Therapeutic landscapes in hospital design: a qualitative assessment by staff and service users of the design of a new mental health inpatient unit. Environment and Planning C: Government and Policy, 25(4), 591-610.

Duxbury, J.A. (2015). The Eileen Skellern Lecture 2014: physical restraint: in defence of the indefensible? Journal of Psychiatric and Mental Health Nursing, 22(2), 92-101.

Fletcher, J., Spittal, M., Brophy, L., Tibble, H., Kinner, S., Elsom, S. and Hamilton, B. (2017). Outcomes of the Victorian Safewards trial in 13 wards: Impact on seclusion rates and fidelity measurement. International Journal of Mental Health Nursing, 26(5), 461-471.

Gaskin, C.J., Elsom, S.J. and Happell, B. (2007). Interventions for reducing the use of seclusion in psychiatric facilities: review of the literature. The British Journal of Psychiatry, 191(4), 298-303.

Gerace A \& Muir Cochrane E (2018). Perceptions of nurses working with psychiatric consumers regarding the elimination of seclusion and restraint in psychiatric inpatient settings and emergency departments: An Australian survey. International Journal of Mental Health Nursing https://doi.org/10.1111/inm.12522

Goulet, M.H., Larue, C. and Dumais, A. (2017). Evaluation of seclusion and restraint reduction programs in mental health: A systematic review. Aggression and violent behavior, 34, 139-146.

Grol, R., Wensing, M., Eccles, M. and Davis, D. eds. (2013). Improving patient care: the implementation of change in health care. Oxford: John Wiley \& Sons. 
Huckshorn, K.A. (2004). Reducing seclusion and restraint use in mental health settings: Core strategies for prevention. Journal of Psychosocial Nursing and Mental Health Services, 42(9), 22-33.

Huckshorn, K.A. (2006). Re-designing state mental health policy to prevent the use of seclusion and restraint. Administration and Policy in Mental Health, 33, 482-491.

Janner, M. (2007). From the inside out: Star Wards-lessons from within acute in-patient wards. Journal of Psychiatric Intensive Care, 3(2), 75.

LeBel, J. (2011). The business case for preventing and reducing restraint and seclusion use. HHS Publication No.(SMA), 11-4632.

LeBel, J.L., Duxbury, J.A., Putkonen, A., Sprague, T., Rae, C. and Sharpe, J. (2014). Multinational experiences in reducing and preventing the use of restraint and seclusion. Journal of Psychosocial Nursing and Mental Health Services, 52(11), 22-29.

Lewis, M., Taylor, K. and Parks, J. (2009). Crisis prevention management: a program to reduce the use of seclusion and restraint in an inpatient mental health setting. Issues in Mental Health Nursing, 30, 159-164.

Lupton, D. (1993). Risk as moral danger: the social and political functions of risk discourse in public health. International Journal of Health Services, 23(3), 425-435.

Muskett, C. (2014). Trauma-informed care in inpatient mental health settings: A review of the literature. International Journal of Mental Health Nursing, 23(1), 51-59.

Murphy, T. and Bennington-Davis, M. (2005). Restraint and seclusion: The model for eliminating their use in healthcare. Marblehead, MA: HC Pro.

NASMHPD Medical Directors Council. (1999). Reducing the use of seclusion and restraint. Alexandria, VA: National Association of State Mental Health Program Directors (NASMHPD), National Technical Assistance Center for State Mental Health Planning (NTAC).

O'Brien, A.J. and Golding, C.G. (2003). Coercion in mental healthcare: the principle of least coercive care. Journal of Psychiatric and Mental Health Nursing, 10(2), 167-173.

Paxton, D. (2009, January 9). The Village Network's experience creating and supporting coercionfree and violence-free treatment environments. Paper presented at the Ohio Department of Mental Health and the Ohio Association of Child Caring Agencies conference, Proactive Positive Interventions, Columbus, $\mathrm{OH}$.

Price, O. and Baker, J. (2012). Key components of de-escalation techniques: A thematic synthesis. International Journal of Mental Health Nursing, 21(4), 310-319. 
Price, O., Baker, J., Bee, P. and Lovell, K. (2015). Learning and performance outcomes of mental health staff training in de-escalation techniques for the management of violence and aggression. The British Journal of Psychiatry, 206(6), 447-455.

Putkonen, A., Kuivalainen, S., Louheranta, O., Repo-Tiihonen, E., Ryynänen, O.P., Kautiainen, H. and Tiihonen, J. (2013). Cluster-randomized controlled trial of reducing seclusion and restraint in secured care of men with schizophrenia. Psychiatric Services, 64(9), 850-855.

Regan, K.M., Curtin, C. and Vorderer, L. (2006). Paradigm shifts in inpatient psychiatric care of children: approaching child-and family-centered care. Journal of Child and Adolescent Psychiatric Nursing, 19(1), 29-40.

Riahi, S., Thomson, G. and Duxbury, J., 2016. An integrative review exploring decisionmaking factors influencing mental health nurses in the use of restraint. Journal of Psychiatric and Mental Health Nursing, 23(2), 116-128.

Robert, G., Cornwell, J., Locock, L., Purushotham, A., Sturmey, G. and Gager, M. (2015). Patients and staff as codesigners of healthcare services. BMJ, 350, 7714.

Rose, N. (1990). Governing the soul: the shaping of the private self. London: Taylor \& Frances/Routledge.

Sanders, K. (2009). The effects of an action plan, staff training, management support and monitoring on restraint use and costs of work-related injuries. Journal of Applied Research in Intellectual Disabilities, 22(2), 216-220.

Smith, G.M., Davis, R.H., Altenor, A., Tran, D.P., Wolfe, K.L., Deegan, J.A. and Bradley, J. (2008). Psychiatric use of unscheduled medications in the Pennsylvania state hospital system: effects of discontinuing the use of PRN orders. Community Mental Health Journal, 44(4), 261-270.

Stame, N. (2004). Theory-based evaluation and types of complexity. Evaluation, 10(1), 5876.

Sweeney, A., Clement, S., Filson, B. and Kennedy, A. (2016). Trauma-informed mental healthcare in the UK: what is it and how can we further its development? Mental Health Review Journal, 21(3), 174-192.

Tambuyzer, E. and Audenhove, C. (2015). Is perceived patient involvement in mental health care associated with satisfaction and empowerment? Health Expectations, 18(4), 516-526. 
Thomann, J. (2009). Factors in restraint reduction in residential treatment facilities for adolescents (Doctoral dissertation, Massachusetts School of Professional Psychology).

Woltmann, E.M., Whitley, R., McHugo, G.J., Brunette, M., Torrey, W.C., Coots, L., Lynde, D. and Drake, R.E. (2008). The role of staff turnover in the implementation of evidence-based practices in mental health care. Psychiatric Services, 59(7), 732-737.

Wright, K., Haigh, K. and McKeown, M. (2007) Reclaiming the humanity in personality Disorder. International Journal of Mental Health Nursing, 16, 236-246. 